\title{
Fentanyl-Induced Brain Hypoxia Triggers Brain Hyperglycemia and Biphasic Changes in Brain Temperature
}

\author{
Ernesto Solis Jr', Keaton T Cameron-Burr', Yavin Shaham' and Eugene A Kiyatkin*,I \\ 'Behavioral Neuroscience Branch, National Institute on Drug Abuse-Intramural Research Program, National Institutes of Health, DHHS, Baltimore, \\ MD, USA
}

\begin{abstract}
Fentanyl is a potent synthetic opioid used extensively in humans for general anesthesia and analgesia. Fentanyl has emerged as a recreational drug, often in combination with heroin, and can result in lethality during overdose. Fentanyl is well characterized as an anesthetic, but the basic physiological effects of fentanyl in the brain when taken as a drug of abuse are largely unknown. We used highspeed amperometry in freely moving rats to examine the effects of intravenous fentanyl at doses within the range of possible human intake (3-40 $\mathrm{\mu g} / \mathrm{kg}$ ) on oxygen and glucose levels in nucleus accumbens (NAc). Fentanyl induced a rapid, dose-dependent decrease in NAc oxygen followed by a more delayed and prolonged increase in NAc glucose. Fentanyl induced similar oxygen decreases in the basolateral amygdala, indicating that brain hypoxia could be a generalized phenomenon. We used oxygen recordings in the subcutaneous space to confirm that fentanyl-induced brain hypoxia results from decreases in blood oxygen levels caused by drug-induced respiratory depression. Temperature recordings in the NAc, muscle, and skin showed that fentanyl induces biphasic changes in brain temperature, with an initial decrease that results primarily from peripheral vasodilation, and a subsequent increase driven by metabolic brain activation. The initial vasodilation appears caused by respiratory depression-induced hypoxia and a subsequent rise in $\mathrm{CO}_{2}$ that drives fentanyl-induced increases in NAc glucose. Together, these data suggest that fentanyl-induced respiratory depression triggers brain hypoxia and subsequent hyperglycemia, both of which precede slower changes in brain temperature and metabolic brain activity.

Neuropsychopharmacology (2018) 43, 810-819; doi:I0.1038/npp.2017.181; published online 20 September 2017
\end{abstract}

\section{INTRODUCTION}

Fentanyl is a potent synthetic opioid drug used extensively in humans for general anesthesia and analgesia (Peng and Sandler, 1999). In recent years, fentanyl has emerged as a recreational drug, often used in combination with heroin (Compton et al, 2016; McLaughlin, 2017). As fentanyl is 20$50 \times$ more potent than heroin (Wade et al, 2015), illicit fentanyl use can result in adverse health effects, including death during overdose (Compton et al, 2016; Suzuki and ElHaddad, 2017). Fentanyl is well characterized as a therapeutic drug (Dahan et al, 2005; Jaffe et al, 1997; Pattinson, 2008; Yeadon and Kitchen, 1989). However, the basic physiological effects of fentanyl in the brain when taken as a drug of abuse are largely unknown.

In addition to unconsciousness and analgesia, fentanyl induces robust respiratory depression (Dahan et al, 2005; Pattinson, 2008; Yeadon and Kitchen, 1989), an effect that poses significant health risks. Although respiratory depression and tissue hypoxia after therapeutic fentanyl administration are well documented, it is unknown how fentanyl

* Correspondence: Dr EA Kiyatkin, Behavioral Neuroscience Branch, NIDA-IRP, DHHS, 333 Cassell Drive, Baltimore, MD 21224, USA. Tel: + | 443740 2844, Fax: + I 44374021 55,

E-mail: ekiyatki@intra.nida.nih.gov

Received I June 2017; revised 20 July 2017; accepted 9 August 2017; accepted article preview online 29 August 2017 quantitatively affects levels of oxygen in the brain within the dose range of possible human consumption. To address this issue, we used high-speed amperometry to examine the effects of intravenous (i.v.) fentanyl on brain oxygen levels in awake, freely moving rats. We supplemented the oxygen measurements with electrochemical evaluations of changes in brain glucose, another critical substrate for metabolic brain activity. Oxygen and glucose enter the brain via arterial blood and their entry into the brain tissue is modulated by neural activity via direct or indirect changes in vascular tone (vasoconstriction/vasodilation) and changes in local cerebral blood flow (Attwell et al, 2010; Lecrux and Hamel, 2011).

Our electrochemical measurements of oxygen and glucose were conducted in the nucleus accumbens (NAc), a region that plays a critical role in mediating the reinforcing effects of addictive drugs (Badiani et al, 2011; Di Chiara, 2002; Wise and Bozarth, 1987). To examine the role of respiratory depression in mediating fentanyl-induced changes in NAc oxygen, we also conducted parallel oxygen recordings in the subcutaneous space, a highly vascularized site with no metabolic activity (Solis et al, 2017a). To examine whether fentanyl-induced changes in NAc oxygen generalize to other brain areas, we conducted oxygen measurements in the basolateral amygdala, a distantly located brain region implicated in conditioned drug effects and drug relapse (Bossert et al, 2013; Everitt and Robbins, 2005). Finally, we examined how fentanyl-induced changes in brain oxygen 
and glucose are related to changes in brain temperature, intrabrain heat production, and skin vascular tone that are basic physiological parameters reflecting brain metabolic activity and heat loss to the external environment. The current study is a follow-up to our recent study, in which we used identical methodologies to examine the effects of heroin on NAc glucose and oxygen (Solis et al, 2017b). In the Discussion section, we compare the effects of fentanyl with those induced by heroin to illustrate similarities and differences between these two opioid drugs that are often used in combination (Compton et al, 2016).

\section{MATERIALS AND METHODS}

\section{Subjects}

Twenty-four male Long-Evans rats (Charles River Laboratories) weighing $460 \pm 40 \mathrm{~g}$ at the time of surgery were used in this study. Rats were individually housed in a climatecontrolled colony maintained on a 12:12 light/dark cycle (lights on at $0600 \mathrm{~h}$ ) with food and water freely available. All procedures were approved by the NIDA-IRP Animal Care and Use Committee and complied with the Guide for the Care and Use of Laboratory Animals (NIH, Publication 86523). Maximal care was taken to minimize the number of experimental animals and any possible discomfort at all stages of the study.

\section{Overview}

This study describes the results of four electrochemical and one thermorecording experiments conducted in awake, freely moving rats. In Exp. 1 and 2, we examined the effects of i.v. fentanyl on extracellular levels of oxygen and glucose in NAc. To clarify possible mechanisms underlying fentanylinduced changes in NAc oxygen, in Exp. 3 we examined the effects of fentanyl on oxygen levels in the subcutaneous space. In Exp. 4 we examined the effects of fentanyl on oxygen levels in basolateral amygdala. Finally, in Exp. 5 we examined the effects of fentanyl on temperatures in NAc, temporal muscle, and skin. As the brain and temporal muscle receive arterial blood from the same carotid artery and thus are equally exposed to heat delivered via blood from the body, changes in NAc-muscle temperature differentials show the source of heat production and serve as a measure of metabolic brain response (Kiyatkin, 2010). Skin temperature is determined by the state of peripheral vessels and its decrease suggest vasoconstriction. However, skin temperature also depends on the temperature of arterial blood inflow. Therefore, skin-muscle temperature differentials exclude this latter contribution, providing a measure of changes in skin vascular tone (Kiyatkin, 2010).

\section{Surgical Preparations}

Surgical procedures for electrochemical experiments are described in detail elsewhere (Kiyatkin and Lenoir, 2012; Solis et al, 2017a). In brief, under general anesthesia (Equithesin, sodium pentobarbital, and chloral hydrate mixture), each rat was unilaterally implanted with a BASi guide cannula (Bioanalytical Systems) into which an electrochemical sensor was later inserted. The target locations were: the right NAc shell $(\mathrm{AP}+1.2 \mathrm{~mm}, \mathrm{ML}$ $\pm 0.8 \mathrm{~mm}$, and DV $+7.3 \mathrm{~mm}$ from the skull surface (Paxinos and Watson, 1998), Exp. 1 and 2), the right basolateral amygdala (AP - $2.8 \mathrm{~mm}$, ML $5.0 \mathrm{~mm}$, and DV $+9.0 \mathrm{~mm}$ from the skull surface, Exp. 4), and the subcutaneous space in the mediofrontal area of the rat's head (Exp. 3). The guide cannula was secured with dental acrylic in a head mount anchored to the skull. During the same surgical procedure, rats were also implanted with a chronic jugular catheter that ran subcutaneously to the head mount and was secured to the same assembly. Rats were allowed a minimum of 5 days of postoperative recovery and at least 3 daily habituation sessions ( $\sim 6 \mathrm{~h}$ each) to the recording environment before experimentation; catheters were flushed daily with $0.2 \mathrm{ml}$ heparinized saline.

Surgical procedures for thermorecording experiment have been described in detail elsewhere (Kiyatkin et al, 2016). In brief, under the same anesthesia, rats were implanted with a jugular catheter and three copper-constantan thermocouple electrodes $(125 \mu \mathrm{m}$ in diameter) in the NAc shell (A-L: 1.2$0.8 \mathrm{~mm}$, depth $7.3 \mathrm{~mm}$ below the skull surface), temporal muscle, and subcutaneously along the nasal ridge with the tip $\sim 15 \mathrm{~mm}$ anterior to the bregma. The probes were secured with dental acrylic to the three stainless steel screws threaded into the skull. We also measured fentanyl-induced changes in locomotor activity using 4 infrared motion detectors (Med Associates).

\section{Electrochemical Detection of Oxygen and Glucose}

In electrochemical experiments, we used commercially produced Pt-based oxygen and glucose sensors (Pinnacle Technology). The principles of electrochemical detection of oxygen and glucose by these sensors, their calibration, and performance have been previously described (Kiyatkin and Wakabayashi, 2015; Solis et al, 2017a) and are presented in the Supplementary Information.

\section{Experimental Procedures}

Electrochemical recordings were performed in single 7-9 h sessions. At the beginning of each session, rats were minimally anesthetized ( $<2 \mathrm{~min})$ with isoflurane and the sensor was inserted either into the NAc (Exp. 1-2), subcutaneous space (Exp. 3), or basolateral amygdala (Exp. 4). The rat was placed in the testing chamber and the sensor connected to the potentiostat via an electrically shielded flexible cable and a multichannel electrical swivel. The injection port of the jugular catheter on the head mount was connected to a plastic catheter extension to allow stress- and cue-free drug delivery from outside the cage. Testing began $\sim 120$ min after insertion of the sensor when baseline currents stabilized.

In Exp. 1-2 (glucose: $n=6$ rats/ 6 sessions; oxygen: $n=8$ rats/10 sessions), we examined changes in oxygen and glucose induced in the NAc by i.v. fentanyl (Fentanyl citrate $50 \mu \mathrm{g} / \mathrm{ml}$; Hospita) at 3, 10, and $40 \mu \mathrm{g} / \mathrm{kg}$ doses (in 0.3, 0.10, and $0.40 \mathrm{ml} ; 0.1 \mathrm{ml}$ over $10 \mathrm{~s}$ ). Rats show consistent selfadministration of fentanyl at $3 \mu \mathrm{g} / \mathrm{kg}$ (Wade et $\mathrm{al}, 2015$ ) and the highest dose used in this study $(40 \mu \mathrm{g} / \mathrm{kg})$, which is lower than the $\mathrm{LD}_{50}$ for rats $(1-3 \mathrm{mg} / \mathrm{kg}$, i.v.; von Gunten et al, 2010), did not cause lethality. Time intervals between 
a

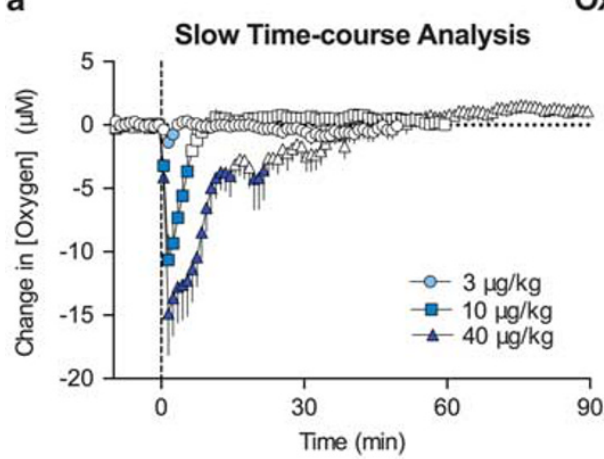

Oxygen

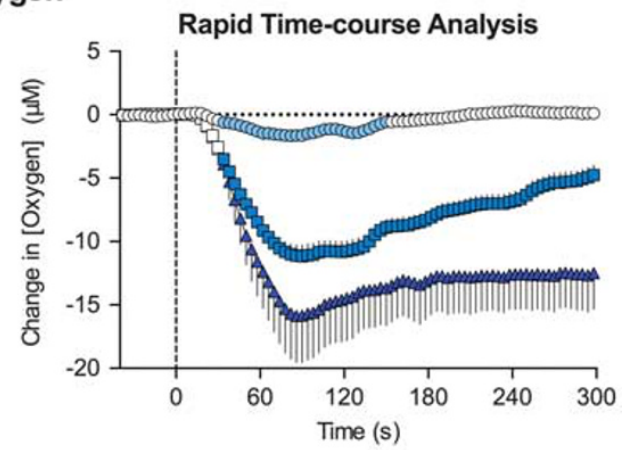

b

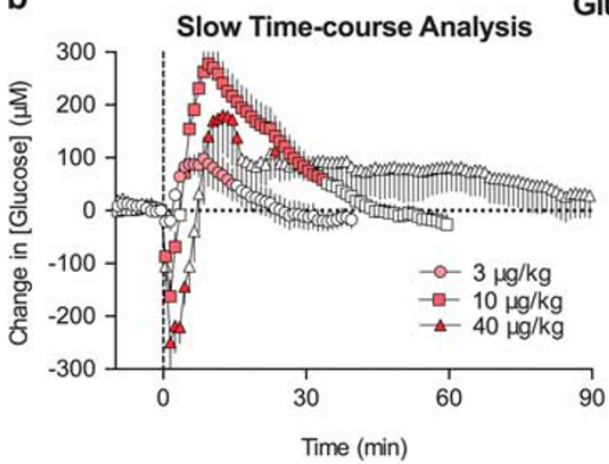

Glucose

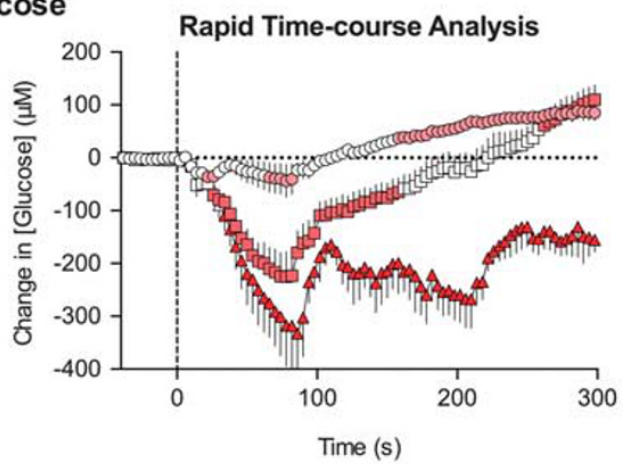

C

Relationships Between Oxygen and Glucose
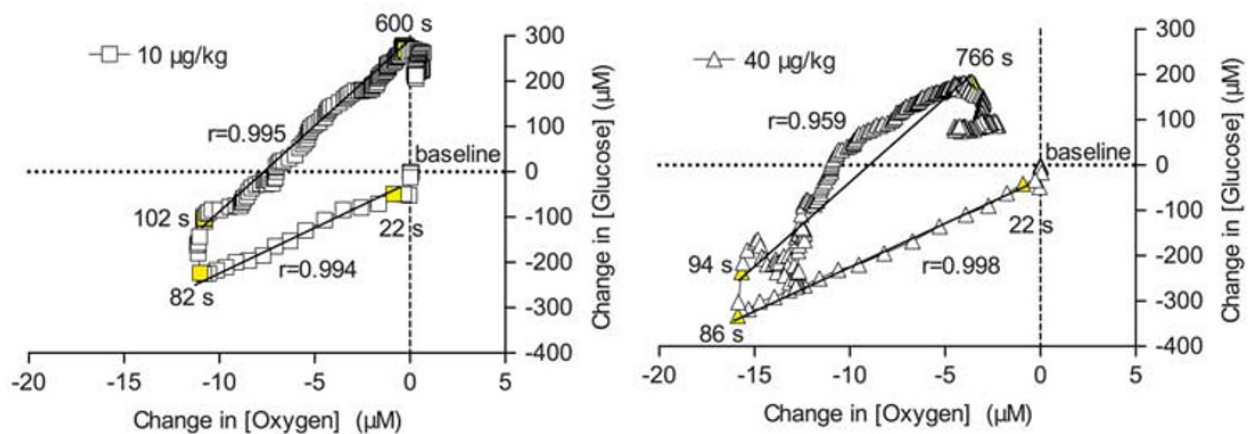

Figure I Mean ( \pm SEM) changes in NAc oxygen (a) and glucose (b) induced by i.v. fentanyl in awake rats. Data are shown with slow (I min; left panel) and rapid ( $4 \mathrm{~s}$ bin; right panel) time resolution as a concentration change $(\mu M)$ relative to preinjection baseline. Values significantly different than preinjection baseline are shown as filled symbols. Time of drug injection $(=0)$ is indicated by the vertical hatched line. (c) The correlation between slow $(I$ min bin) and rapid (4 s bin) changes in oxygen and glucose induced by fentanyl at the 10 and $40 \mathrm{\mu g} / \mathrm{kg}$ doses is shown. The value immediately preceding heroin injection $(0$ for both oxygen and glucose) was used as baseline. The period assessed for correlation is shown as the period between yellow-colored symbols with numbers. A line of best fit is shown with the correlation coefficient $(r)$. For additional details see the text.

injections varied between 60 and 120 min depending on the dose, and represented sufficient time for restoration of preinjection baselines. In Exp. 3-4, in which oxygen recordings were conducted in the subcutaneous space $(n=4)$ and amygdala $(n=6)$, the rats were exposed to three injections of fentanyl (two at 10 and one at $40 \mu \mathrm{g} / \mathrm{kg}$ ). In all electrochemical experiments, fentanyl was injected at ascending doses. Similarly, in thermorecording experiment (Exp. 5), rats $(n=6)$ received 3 fentanyl injections: two at $10 \mu \mathrm{g} / \mathrm{kg}$ and one at $40 \mu \mathrm{g} / \mathrm{kg}$. At the end of recording sessions, rats were anesthetized with Equithesin (i.v. $1.0 \mathrm{ml}$ ), disconnected from the instrument, sensors were carefully removed and, in the case of glucose, recalibrated. Typically, the rats underwent only one recording session. At the end of the experiments, they were deeply anesthetized, their brains were removed, stored in $10 \%$ formalin, and sectioned for verification of sensor placement using a rat stereotaxic atlas; the brain sections were also assessed for possible gross anatomical damage (see Supplementary Figure S1 in Supplementary Information). In several rats, electrochemical recordings with oxygen sensors were repeated 2 to 4 days later.

\section{Data Analysis}

Electrochemical data were sampled with $1 \mathrm{~Hz}$ using the PAL software utility (Version 1.5.0, Pinnacle Technology) and analyzed with two time resolutions (slow, with a 1 min time 
bin and rapid, with a $4 \mathrm{~s}$ bin). Electrochemical data were first analyzed as raw currents. Because individual sensors slightly differed in their background current and substrate sensitivity in vitro, currents were then transformed into concentrations and are represented as relative concentration changes, taking a prestimulus baseline current as 0 . One-way repeated measure ANOVAs (followed by Fisher's PLSD post hoc tests) were used to evaluate the statistical significance of druginduced changes in oxygen and glucose concentrations, and the temperature parameters. We also used time-dependent correlation analyses to examine the relationships between oxygen, glucose, changes in temperature, and temperature differentials.

\section{RESULTS}

\section{Changes in NAc Oxygen and Glucose Induced by Fentanyl}

Oxygen. At slow, $1 \mathrm{~min}$ time resolution, fentanyl monophasically decreased NAc oxygen levels $\left(\mathrm{F}_{5,70}=11.8\right.$, $\mathrm{F}_{12,204}=15.6$, and $\mathrm{F}_{9,414}=12.0$ for 3,10 , and $40 \mu \mathrm{g} / \mathrm{kg}$, respectively; $p<0.001$; Figure 1a). The decrease was rapid, reaching nadir $1.5 \mathrm{~min}$ after injection onset, and its magnitude was dose dependent. Decreases were weak and transient at $3 \mu \mathrm{g} / \mathrm{kg}$, larger in amplitude and duration at $10 \mu \mathrm{g} / \mathrm{kg}$, and most pronounced and prolonged at $40 \mu \mathrm{g} / \mathrm{kg}$. When calculated as percent change with respect to basal oxygen levels, the mean magnitude of decrease from baseline was $-7.4,-46.5$, and $-64.1 \%$ for 3,10 , and $40 \mu \mathrm{g} / \mathrm{kg}$, respectively. When analyzed at rapid, $4 \mathrm{~s}$ time resolution, we found that at each dose oxygen levels began to decrease with an $\sim 30 \mathrm{~s}$ latency, reaching nadir at $\sim 90 \mathrm{~s}$ (Figure 1a). Fentanyl-induced oxygen decreases were shortest in duration at the $3 \mu \mathrm{g} / \mathrm{kg}$ dose and became progressively stronger at 10 and $40 \mu \mathrm{g} / \mathrm{kg}$.

Glucose. Fentanyl also induced significant changes in NAc glucose levels $\left(\mathrm{F}_{17,442}=4.3, \mathrm{~F}_{15,690}=23.7\right.$, and $\mathrm{F}_{5,450}=3.3$ for 3,10 , and $40 \mu \mathrm{g} / \mathrm{kg}$, respectively; $p<0.001$; Figure $1 \mathrm{~b}$ ). In contrast to oxygen, the effect of fentanyl on glucose was biphasic, showing a down-up fluctuation that became more pronounced with dose increase. At slow temporal resolution $(1 \mathrm{~min})$, only a moderate increase was seen after the $3 \mu \mathrm{g} / \mathrm{kg}$ dose, but the response became biphasic at 10 and $40 \mu \mathrm{g} / \mathrm{kg}$. When data were calculated as percent change with respect to basal glucose levels, the mean magnitude of change was $-2.5 /+13.1 \%,-22.6 /+37.5 \%$, and $-38.2 /+26.1 \%$ for 3,10 , and $40 \mu \mathrm{g} / \mathrm{kg}$, respectively. Biphasic NAc glucose dynamics were evident at all doses when data were analyzed with high temporal resolution ( $4 \mathrm{~s}$, Figure $1 \mathrm{~b}$ ). The initial decrease in glucose became progressively larger with increased drug dose, and had an $\sim 30 \mathrm{~s}$ latency, like that seen with the oxygen decrease.

Next, we examined the relationship between fentanylinduced changes in NAc oxygen and glucose by analyzing how glucose levels change with respect to changes in oxygen levels after drug injection. When calculated for the entire duration of the drug effect, fentanyl-induced changes in oxygen and glucose highly correlated $(r=0.854$ and $r=0.796$ for 10 and $40 \mu \mathrm{g}$, respectively). When data were correlated within the time interval of maximal drug effect, we found that at $10 \mu \mathrm{g} / \mathrm{kg}$ both parameters began to change with a similar latency $(\sim 22 \mathrm{~s})$, and showed high correlation $(r=0.994)$ up to $\sim 82 \mathrm{~s}$, when both glucose and oxygen levels reached their respective nadirs. Then, both parameters began to increase, showing a similarly high correlation $(r=0.995)$ that was maintained from 102 to $600 \mathrm{~s}$ after injection onset, when oxygen levels returned to their baseline and glucose levels reached their peak. Then, glucose levels began to descend to baseline as oxygen levels remained stable at baseline levels. Similar relationships were observed at the $40 \mu \mathrm{g} / \mathrm{kg}$ dose (Figure 1c). Both parameters had similar latencies and began to decrease at $\sim 22 \mathrm{~s}$. Oxygen and glucose showed a high correlation $(r=0.998)$ over $22-82 \mathrm{~s}$, and a similar correlation $(r=0.959)$ during the period when oxygen levels rebound to their baseline and glucose levels rise to their peak (94-766s).

\section{Respiratory Depression and Decrease in Blood Oxygen as the Cause of Brain Hypoxia}

To explore the role that fentanyl-induced respiratory depression and the subsequent decrease in blood oxygen levels play in brain hypoxia, we conducted oxygen measurements in the subcutaneous space, a highly vascularized area with minimal or no metabolic activity. Oxygen measurements acquired from this location serve as a useful proxy for arterial blood levels (Moon et al, 2013) that are technically difficult to directly measure with high temporal resolution in freely moving rats.

Fentanyl induced dose-dependent decreases in oxygen levels in the subcutaneous space $\left(\mathrm{F}_{7,322}=13.5\right.$ and $\mathrm{F}_{3,138}=11.4$ for 10 and $40 \mu \mathrm{g} / \mathrm{kg}$, respectively; $p<0.001$; Figure 2). These decreases were nearly identical in magnitude and duration to those seen in the NAc (Figure 2a). Rapid time-course analysis revealed almost identical oxygen changes in onset latency and magnitude between the subcutaneous space and the NAc.

\section{Generality of Oxygen Response in the Brain: NAc and Amygdala}

To determine whether fentanyl-induced changes in NAc oxygen generalize to other brain areas, we examined the effects of fentanyl in the basolateral amygdala. Like in the NAc, fentanyl decreased oxygen levels in the amygdala $\left(\mathrm{F}_{5,55}=11.7\right.$ and $\mathrm{F}_{2,82}=4.6$ for 10 and $40 \mu \mathrm{g} / \mathrm{kg} ; p<0.001$; Figure $3 \mathrm{a})$. In both regions, the decreases in oxygen occurred with similar onset latencies, reached nadir, and returned to the preinjection baseline at comparable times. Furthermore, basal oxygen levels in both regions evaluated based on sensor sensitivity were similar (amygdala: $19.5 \pm 3.0 \mu \mathrm{M}, \mathrm{NAc}$ : $22.9 \pm 4.1 \mu \mathrm{M})$.

\section{Changes in Temperature and Locomotor Activity Induced by Fentanyl: Relationships between Drug- Induced Changes in Oxygen and Glucose, Metabolic Brain Activation, and Vascular Response}

Fentanyl at both doses significantly changed NAc, muscle, and skin temperatures (Figure $4 \mathrm{a}, 10 \mu \mathrm{g} / \mathrm{kg}: \mathrm{F}_{8,224}=2.9,2.7$, and 3.4 for NAc, muscle, and skin, respectively; $p<0.001$; $40 \mu \mathrm{g} / \mathrm{kg}: \mathrm{F}_{8,488}=12.9,15.7$, and 10.6 for NAc, muscle, and 
a

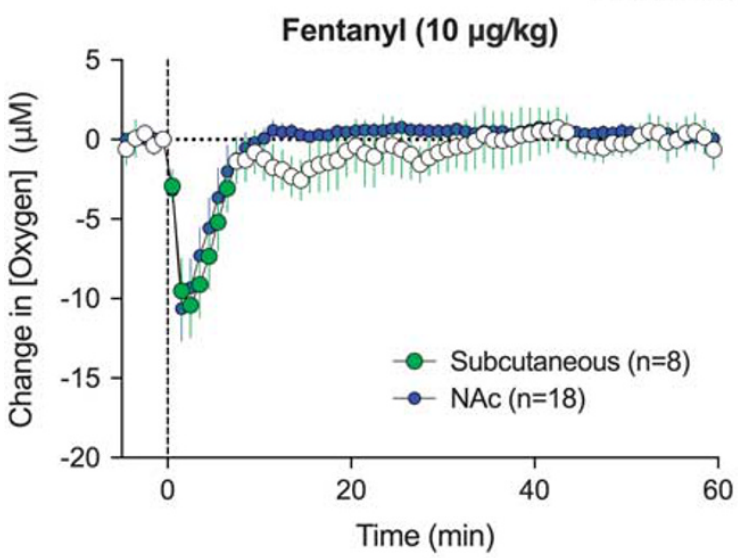

Fentanyl $(40 \mu \mathrm{g} / \mathrm{kg})$

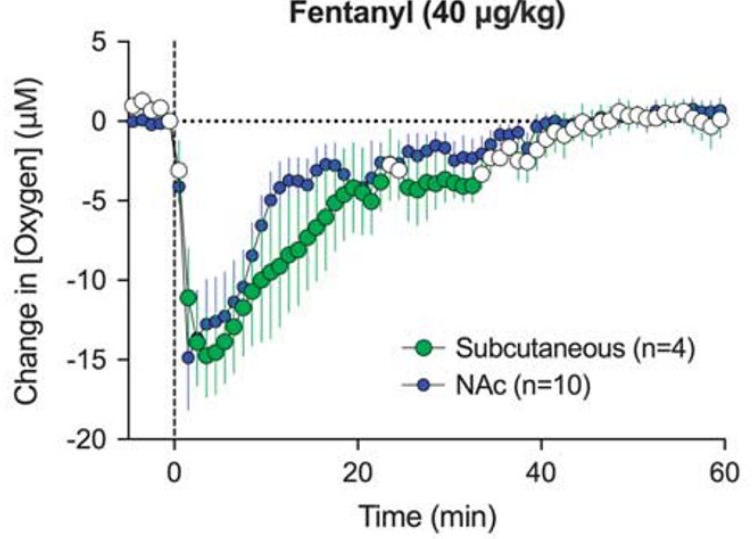

b Rapid Time-course Analysis
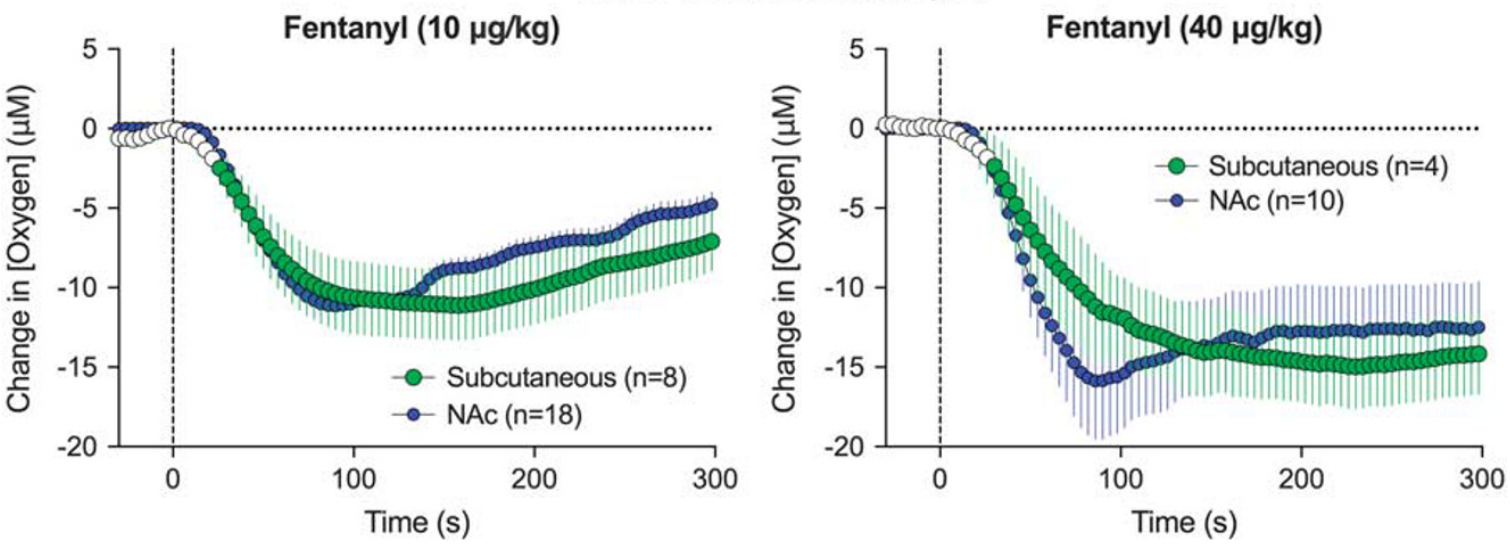

Figure 2 Mean $( \pm$ SEM) changes in oxygen levels in subcutaneous space induced by i.v. fentanyl. Data were analyzed with slow (a, I min bin) and rapid (b, $4 \mathrm{~s}$ bin) time resolution are shown. Values significantly different than preinjection baseline are shown as filled symbols. Data obtained in the NAc are superimposed on each graph for comparison.

skin, respectively; $p<0.001)$ and induced a transient decrease in locomotor activity (Figure $4 \mathrm{~b}$ ). The effects were dose dependent and showed a biphasic pattern, with the initial decrease followed by an increase above baseline. At the $10 \mu \mathrm{g} / \mathrm{kg}$ dose, changes were relatively weak, and the initial temperature decrease was the dominant effect. Both the initial decrease and subsequent increase were stronger at the $40 \mu \mathrm{g} / \mathrm{kg}$ dose, and the temperature increase above baseline following the initial temperature decrease became the dominant effect.

Next, we used correlation analysis to examine the relationships between fentanyl-induced changes in temperature, oxygen, and glucose. At $10 \mu \mathrm{g} / \mathrm{kg}$, we found that oxygen changes were independent of changes in brain and muscle temperatures $(r=0.26$ and $0.15, p>0.05)$ but negatively correlated with skin temperature $(r=-0.49, p<0.01)$. In contrast, significant correlation was found for changes in glucose levels and temperatures in all recording locations $(r=-0.51,-0.75$, and -0.79 for NAc, muscle, and skin; $p<0.001)$. The relationships between temperature, oxygen, and glucose were different for fentanyl at the $40 \mu \mathrm{g} / \mathrm{kg}$ dose. In this case, oxygen levels significantly correlated with all temperatures $(r=0.70,0.64$, and 0.50 for NAc, muscle, and skin, respectively; $p<0.001$ ) but changes in glucose levels were independent of changes in temperature $(r=-0.06$, -0.12 , and $-0.24, p>0.05$ ).

Two calculated parameters, NAc-muscle and skin-muscle differentials, allow us to evaluate whether changes in temperature result from changes in intrabrain heat production (because of metabolic activity) and/or skin heat loss or retention (because of changes in vascular tone). We found that fentanyl increased skin-muscle differentials at both 10 and $40 \mu \mathrm{g} / \mathrm{kg} \quad\left(\mathrm{F}_{8,224}=3.4\right.$ and $\mathrm{F}_{8,368}=3.8$, respectively; $p<0.001$ ), suggesting rapid skin vasodilation that precedes and likely mediates the initial hypothermic response (Figure 5). Fentanyl also increased the NAc-muscle differentials $\left(10 \mu \mathrm{g} / \mathrm{kg}: \mathrm{F}_{8,224}=3.3\right.$ and $40 \mu \mathrm{g} / \mathrm{kg}: \mathrm{F}_{8,368}=4.6$; $p<0.001$ ), suggesting metabolic brain activation. However, this effect appeared with 15-20 min latency, suggesting a role for metabolic brain activation in mediating the second, hyperthermic phase of the temperature response.

Changes in both oxygen and glucose were not correlated with the NAc-muscle differential $(10 \mu \mathrm{g} / \mathrm{kg}: r=0.23$ and 0.11 , respectively; $40 \mu \mathrm{g} / \mathrm{kg}: r=0.28$ and 0.29 ), suggesting their independence. In contrast, changes in oxygen showed strong negative correlation with changes in the skin-muscle differential $(r=-0.76$ and -0.87 for 10 and $40 \mu \mathrm{g} / \mathrm{kg})$ but 
a

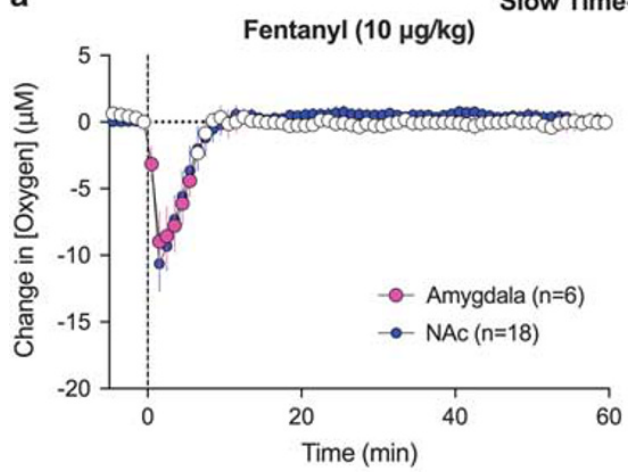

Slow Time-Course Analysis

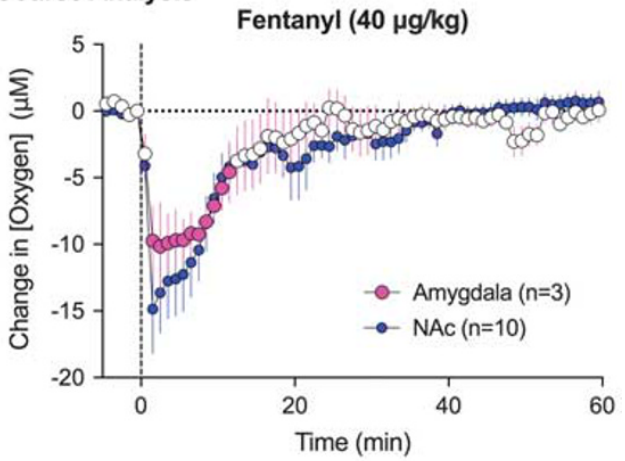

b Rapid Time-Course Analysis
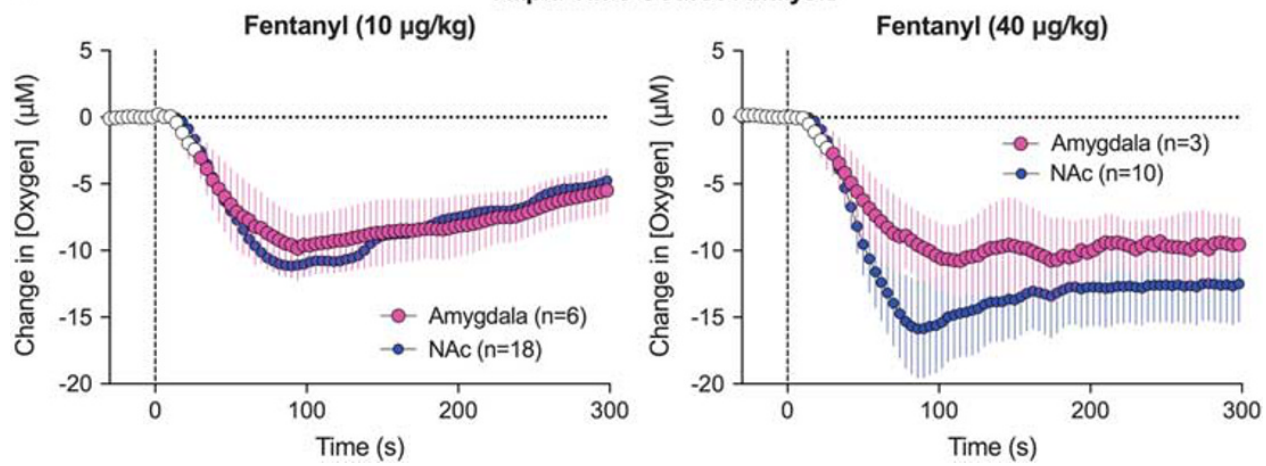

Figure 3 Mean $( \pm$ SEM) changes in oxygen levels in the basolateral amygdala and NAc induced by i.v. fentanyl. Data are shown with slow (I min; a) and rapid (4s bin; b) time resolution as a concentration change $(\mu M)$ relative to preinjection baseline. Values significantly different from preinjection baseline are shown as filled symbols; $n=$ number of averaged tests.

this parameter did not correlate with fentanyl-induced changes in glucose $(p>0.05)$.

\section{DISCUSSION}

There are four main findings in this study. First, fentanyl induced a rapid decrease in NAc oxygen followed by a delayed and prolonged increase in NAc glucose. Second, fentanyl induced similar oxygen decreases in the basolateral amygdala, suggesting that brain hypoxia might be a generalized phenomenon. Third, oxygen recordings in the subcutaneous space confirm that fentanyl-induced brain hypoxia results from decreases in blood oxygen levels caused by drug-induced respiratory depression. Fourth, fentanyl induced biphasic changes in brain temperature, with an initial decrease that results primarily from peripheral vasodilation, and a subsequent increase driven by metabolic brain activation. Together, our data suggest fentanyl-induced respiratory depression as a trigger for rapid brain hypoxia and subsequent hyperglycemia, both of which preceded slower changes in brain temperature and metabolic brain activity.

\section{Fentanyl-Induced Changes in Brain Oxygen and Glucose and Their Underlying Mechanisms}

Fentanyl dose-dependently decreased NAc oxygen levels. The strength $(-65 \%)$ and duration $(20-30 \mathrm{~min})$ of oxygen decrease observed at the $40 \mu \mathrm{g} / \mathrm{kg}$ dose indicate that fentanyl induces robust brain hypoxia. Our oxygen recordings in the subcutaneous space confirmed that fentanyl-induced brain hypoxia results from the drug-induced decrease in blood oxygen levels that is driven by respiratory depression.

In contrast to monophasic decreases in oxygen, fentanylinduced changes in NAc glucose were biphasic. At the $3 \mu \mathrm{g} /$ $\mathrm{kg}$ dose, we observed a rapid but weak decrease in glucose followed by a modest increase. However, the initial decrease became more pronounced at higher doses and at $40 \mu \mathrm{g} / \mathrm{kg}$ it became larger in magnitude than the subsequent increase. Fentanyl-induced changes in glucose strongly correlated with changes in oxygen, but the dynamics of correlation within the time course of drug effect were complex and twocomponent in nature. The initial decrease in glucose correlated with the oxygen decrease and the subsequent rise in glucose correlated with the rise in oxygen from its nadir to baseline. These data indicate that fentanyl-induced changes in glucose depend on drug-induced brain hypoxia but the mechanisms underlying the two-phasic change in glucose remain unclear.

Proximal neural activation that results in local vasodilation is regarded as the primary mechanism underlying enhanced glucose entry into brain tissue under physiological conditions (Attwell et al, 2010; Fellows et al, 1992; Silver and Erecinska, 1994). However, our thermorecording findings do not show brain activation during the interval of glucose rise, suggesting that this mechanism does not mediate the 

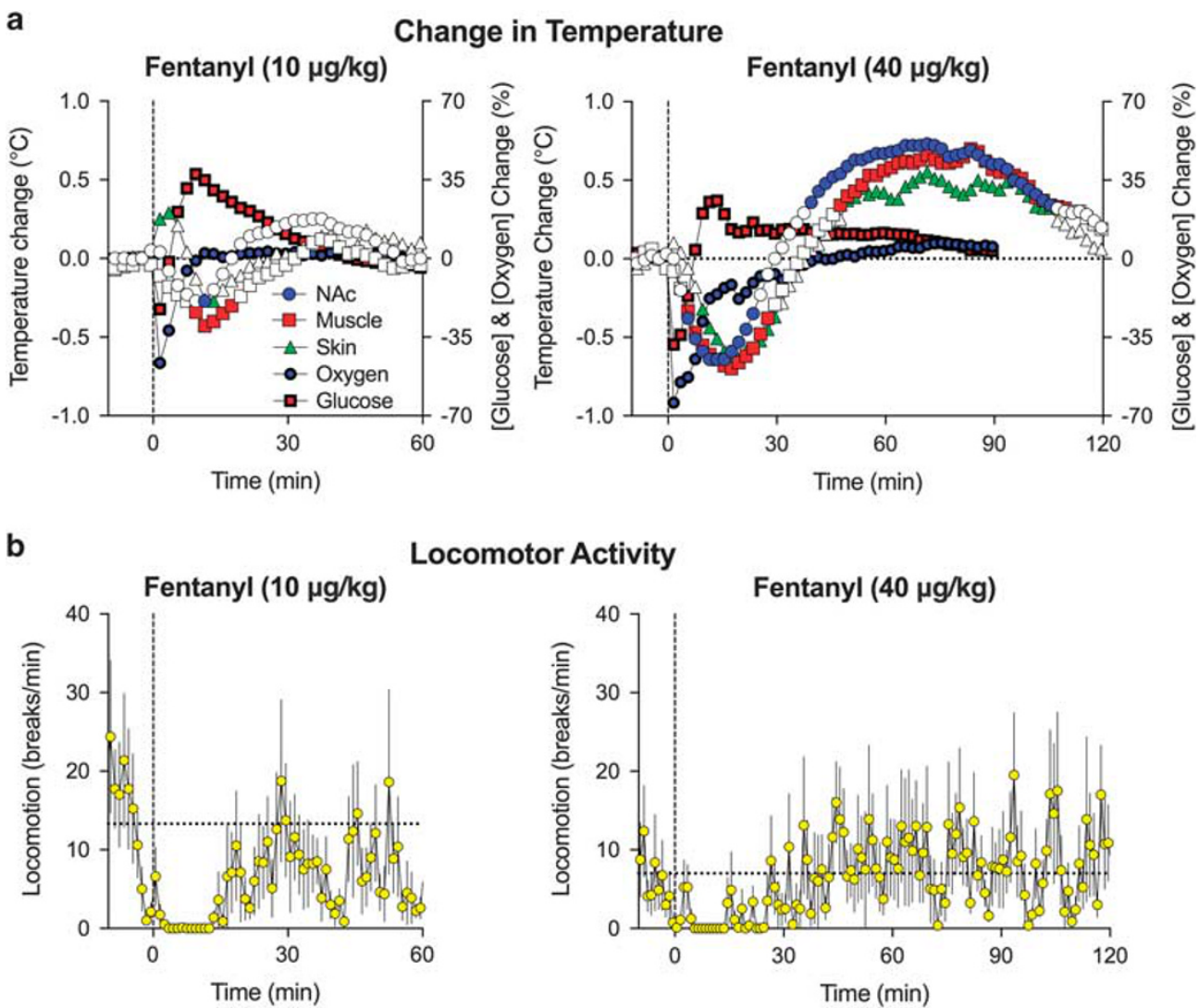

Locomotor Activity

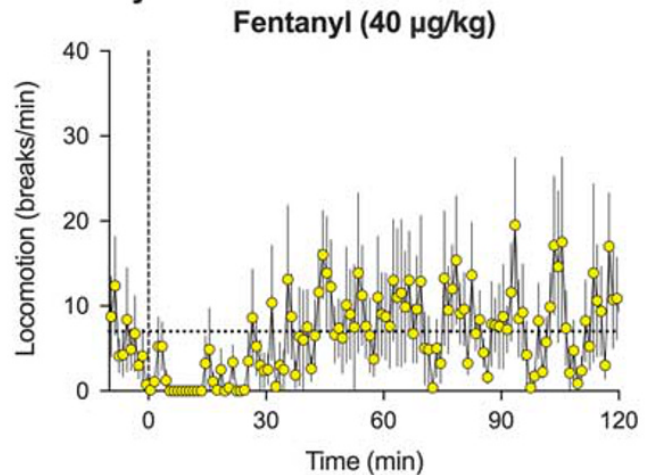

Figure 4 Mean $( \pm$ SEM) changes in NAc, muscle, and skin temperature (a) and locomotor activity (b) induced by i.v. fentanyl. Values significantly different from baseline are shown as filled symbols. For clarity, SEs are not shown for temperature parameters. Changes in NAc oxygen and glucose shown as percent change of preinjection baseline are superimposed on temperature graphs. For additional details see text.

fentanyl-induced glucose increase. It is also unlikely that fentanyl affects blood glucose levels, suggesting the twocomponent vascular response as the possible cause of the fentanyl-induced biphasic glucose change. As respiratory depression results in the accumulation of $\mathrm{CO}_{2}$, a strong vasodilator in the bloodstream and brain tissue (Battisti-Charbonney et al, 2011; Schmidt and Kety, 1947), fentanyl-induced acute hypoxia should cause local vasodilation that, in turn, likely facilitates the rise in NAc glucose. Accumulation of $\mathrm{CO}_{2}$ could also drive peripheral vasodilation, as suggested by our thermorecording experiments.

It is more difficult to explain why glucose initially rapidly decreased after fentanyl injection. One possibility is that fentanyl induces a drop in arterial blood pressure that results in decreased perfusion pressure and subsequent decrease in glucose entry into brain tissue. The i.v. fentanyl induces hypotension, with decreases in arterial blood pressure up to 30-50 $\mathrm{mm} \mathrm{Hg}$ (Laubie et al, 1974) because of decreased sympathetic output and peripheral vasodilation, a mechanism confirmed by our thermorecording experiments. As oxygen enters the brain tissue via passive diffusion, decreased perfusion pressure could also amplify the fentanyl-induced oxygen decrease. It is also possible that fentanyl-induced peripheral vasodilation is accompanied by central vasoconstriction that could explain the initial decrease in NAc glucose levels.

In our study, fentanyl-induced changes in oxygen were similar in the NAc and basolateral amygdala, suggesting that they may represent a generalized phenomenon. Recordings from additional structures are necessary to confirm this idea, but it is likely that this is the case because the decrease in brain oxygen depends on the oxygen drop in arterial blood and all brain structures have a good arterial supply.

\section{Fentanyl-Induced Changes in Brain Oxygen and Glucose: Relationships with Brain Temperature, Metabolic Activation, and Vascular Response}

Our thermorecording experiments allowed us to determine the relationship between fentanyl-induced changes in oxygen and glucose and changes in metabolic brain activity and peripheral vascular tone. We found that fentanyl induces a dose-dependent, biphasic down-up change in brain temperature. The initial hypothermia was evident during the first $30 \mathrm{~min}$ after injection, the time interval when the major changes in oxygen and glucose occur. By calculating skinmuscle temperature differentials, we showed that the temperature decrease is primarily the result of enhanced heat dissipation because of peripheral vasodilation, a rapid change that correlated with decreased NAc oxygen levels. As suggested by changes in NAc-muscle temperature differentials, fentanyl also induced intrabrain heat production because of metabolic neural activation. This effect, however, occurred 15-60 min after injection when drug-induced sedation was no longer observed and both oxygen and glucose began to return to baseline levels. Therefore, 

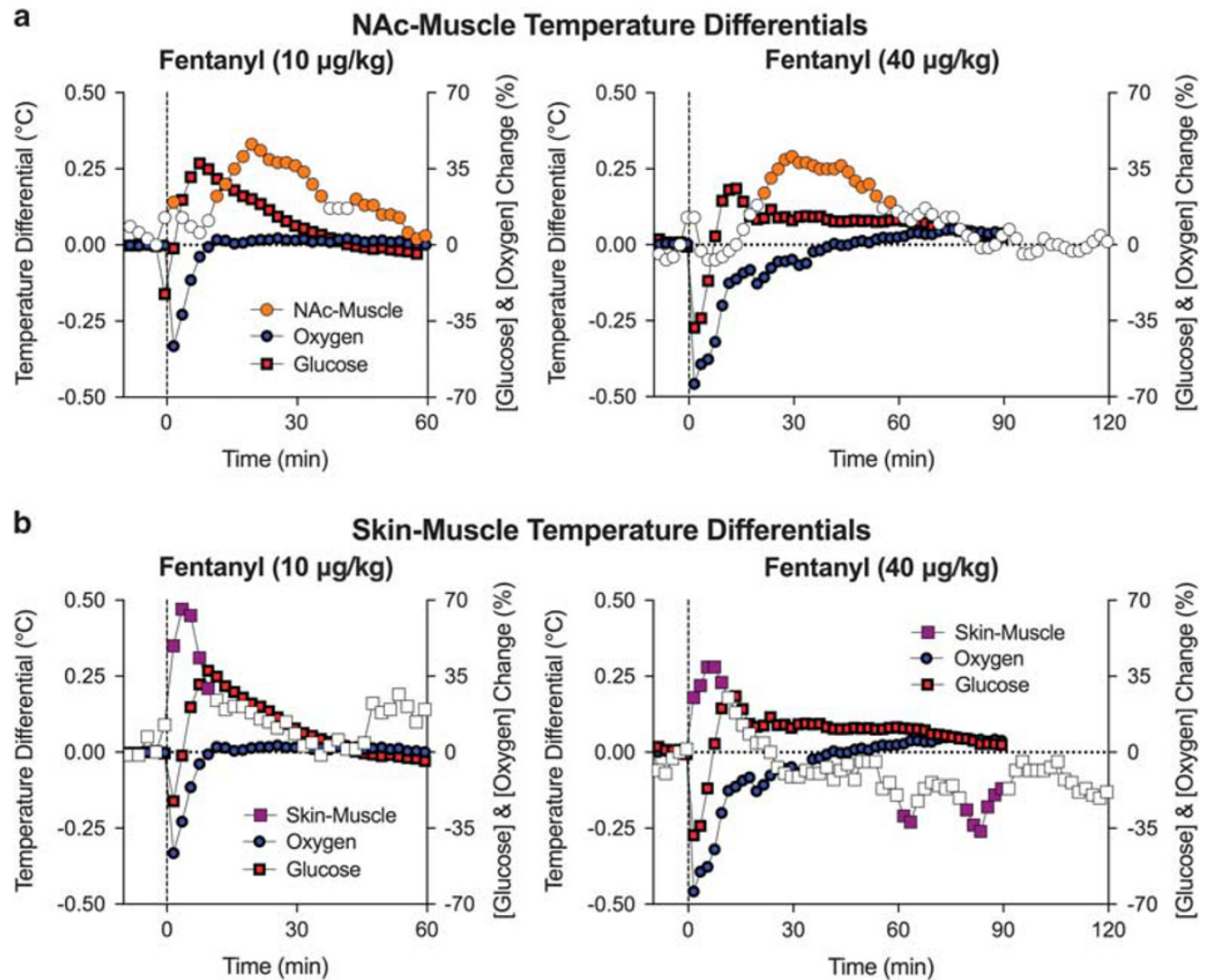

Figure 5 Mean $( \pm$ SEM) changes in NAc-muscle (a) and skin-muscle temperature differentials (b) induced by i.v. fentanyl. Values significantly different from baseline are shown as filled symbols. For clarity, SEs are not shown for temperature differentials. Changes in NAc oxygen and glucose shown as percent change of preinjection baseline are superimposed on temperature graphs. For additional details see text.

fentanyl-induced changes in oxygen and glucose seem independent of metabolic brain activation. The metabolic brain activation that appears after drug-induced sedation could be a compensatory response to changes in brain metabolism after brain hypoxia resulting from drug-induced respiratory depression.

Functional magnetic resonance imagining (fMRI) is a noninvasive procedure that measures brain activity by detecting changes associated with blood flow (Attwell and Iadecola, 2002; Hall et al, 2016). Although commonly used as a surrogate of neuronal activity, blood-oxygenation leveldependent (BOLD) signals reflect changes in brain blood oxygenation. As fentanyl robustly decreased brain oxygen, it should also decrease BOLD signals. Consistent with this notion, BOLD signals in rats strongly decreased after an i.v. injection of heroin rats (Xu et al, 2000). Importantly, this decrease was global, evident in different brain structures, and the decreased oxygenation subsided when the rats were placed under artificial respiration. However, results from fMRI studies in rats should be interpreted with caution because they lack necessary spatial resolution, and require anesthesia or strict head restraint that both affect neuronal activity (Austin et al, 2005; Windels and Kiyatkin, 2006), brain metabolism, and the state of cerebral vessels (Baughman et al, 1986; Nakao et al, 2001), as well as intracerebral levels of oxygen and glucose (Bola and Kiyatkin, 2016; Ciobanu et al, 2012; Liu et al, 1997).

\section{Comparison with Heroin}

Fentanyl's effects on brain oxygen and glucose mirror the effects of heroin with some important differences (Solis et al, 2017b). Direct comparison of the two drugs should be made with caution because the two drugs have different potencies and pharmacokinetics (Wade et al, 2015). Both heroin and fentanyl depress respiration and induce brain hypoxia followed by hyperglycemia. However, at doses $10 \times$ higher than fentanyl (100 vs $10 \mu \mathrm{g} / \mathrm{kg})$, heroin induces twofold weaker decreases in NAc oxygen, suggesting that fentanyl is $\sim 20$-fold stronger in its ability to inhibit respiration. However, heroin induces larger and more prolonged increases in NAc glucose, with less evident postinjection decreases than fentanyl. In contrast to fentanyl, which at all tested doses induced a biphasic down-up change in brain temperature driven by initial vasodilation and delayed metabolic activation, heroin at low doses induces a monophasic temperature increase driven by vasoconstriction. However, at higher doses, like fentanyl, heroin causes biphasic changes in brain temperature that results from initial vasodilation followed by vasoconstriction. Although both drugs also induced metabolic brain activation with significant latency following major changes in oxygen and glucose, the magnitude of activation was greater for fentanyl. 


\section{Conclusions and Functional Implications}

We showed that i.v. fentanyl at doses within the range of possible human use induces rapid, robust, and dosedependent brain hypoxia that results from drug-induced respiratory depression and decreases in blood oxygen levels. Respiratory depression and associated brain hypoxia appear to drive subsequent increases in brain glucose that result primarily from accumulation of $\mathrm{CO}_{2}$ and subsequent cerebral vasodilation. Fentanyl is at least 20 -fold more potent than heroin in causing brain hypoxia. Thus, its consumption, either by mistaking it for heroin or as a contaminant of 'street heroin,' could result in strong brain hypoxia, with possible lethality. Fentanyl also induces an acute state of unconsciousness, fully blocking motor responses to external and internal stimuli (Jaffe et al, 1997). Therefore, the inability to control one's body, particularly to maintain respiratory function, could increase the likelihood of asphyxiation after fentanyl intake. The rapid effects of fentanyl on brain oxygen shown in this study greatly limit the efficacy of therapeutic interventions by opioid antagonists to block the action of fentanyl, revive respiratory activity, and save the life of intoxicated individuals. To be effective, the treatment should be delivered as quickly as possible after fentanyl intake.

Other considerations are likely important in the translation of our rat data to humans. First, fentanyl is an exceptionally strong and rapidly acting drug, with significant hypoxic effects reported in human patients at the $3-7 \mu \mathrm{g} / \mathrm{kg}$ dose range (Dahan et al, 2005). These doses are much smaller than traditional estimates of $\mathrm{LD}_{50}$ in rats $(1-3 \mathrm{mg} / \mathrm{kg})$ but closer to values reported in monkeys $(30 \mu \mathrm{g} / \mathrm{kg}$ ) (von Gunten et al, 2010). It also appears that rats have greater resistance to hypoxia than humans. The duration of apnea in isoflurane-anesthetized rats after i.v. fentanyl $(8 \mu \mathrm{g} / \mathrm{kg})$ could reach $\sim 6$ min (Willette et al, 1987) that would likely result in lethality if apnea of such duration were to occur in humans. Therefore, potentially lethal doses of fentanyl in humans are likely much lower than those in rats.

\section{FUNDING AND DISCLOSURE}

The authors declare no conflict of interest.

\section{ACKNOWLEDGMENTS}

These studies were supported by the Intramural Research Program, NIH.

\section{REFERENCES}

Attwell D, Buchan AM, Charpak S, Lauritzen M, Macvicar BA, Newman EA (2010). Glial and neuronal control of brain blood flow. Nature 468: 232-243.

Attwell D, Iadecola C (2002). The neural basis of functional brain imaging signals. Trends Neurosci 25: 621-625.

Austin VC, Blamire AM, Sharp T, Styles P, Matthews PM, Sibson NR (2005). Confounding effects of anesthesia on functional activation in rodent brain: a study of halothane ad alphachloralose anesthesia. Neuroimage 24: 92-100.
Badiani A, Belin D, Epstein D, Calu D, Shaham Y (2011). Opiate versus psychostimulant addiction: the differences do matter. Nat Rev Neurosci 12: 685-700.

Baughman VL, Hoffman WE, Miletich DJ, Albreacht RF (1986). Effects of pentobarbital on cerebral blood flow and metabolism in young and aged rats. Anesthesiology 65: 500-505.

Battisti-Charbonney A, Fisher JA, Duffin J (2011). Respiratory, cerebrovascular and cardiovascular responses to isocapnic hypoxia. Respir Physiol Neurobiol 179: 259-268.

Bola RA, Kiyatkin EA (2016). Robust brain hyperglycemia during general anesthesia: relationships with metabolic brain inhibition and vasodilation. Front Physiol 7: 39.

Bossert JM, Marchant NJ, Calu DJ, Shaham Y (2013). The reinstatement model of drug relapse: recent neurobiological findings, emerging research topics, and translational research. Psychopharmacology (Berl) 229: 453-476.

Ciobanu L, Reynaud O, Uhrig L, Jarraya B, Le Bihan D (2012). Effects of anesthetic agents on blood oxygenation levels revealed with ultra-high field MRI. PLoS ONE 7: e32645.

Compton WM, Jones CM, Baldwin GT (2016). Relationship between nonmedical prescription-opioid use and heroin use. $N$ Engl J Med 374: 154-163.

Dahan A, Yassen A, Bijl H, Romberg R, Sarton E, Teppema L et al (2005). Comparison of the respiratory effects of intravenous buprenorphine and fentanyl in humans and rats. Br J Anaesth 94: 825-834.

Di Chiara G (2002). Nucleus accumbens shell and core dopamine: differential role in behavior and addiction. Behav Brain Res 137: $75-114$.

Everitt BJ, Robbins TW (2005). Neural systems of reinforcement for drug addiction: from actions to habits to compulsion. Nat Neurosci 8: 1481-1489.

Fellows LK, Boutelle MG, Fillenz M (1992). Extracellular brain glucose levels reflect local neuronal activity: a microdialysis study in awake, freely moving rats. J Neurochem 59: 2141-2147.

Hall CN, Howarth C, Kurth-Nelson Z, Mishra A (2016). Interpreting BOLD: toward a dialogue between cognitive and cellular neuroscience. Philos Trans R Soc Lond B Biol Sci 371: 20150348.

Jaffe JH, Knapp CM, Ciraulo DA (1997). Opiates: clinical aspects. In: Lowinson JH, Ruiz P, Millman RB, Langrod JG (eds). Substance Abuse, 3rd edn. Williams \& Wilkins: Baltimore, pp 158-166.

Kiyatkin EA (2010). Brain temperature homeostasis: physiological fluctuations and pathological shifts. Front Biosci (Landmark Ed) 15: 73-92.

Kiyatkin EA, Lenoir M (2012). Rapid fluctuations in extracellular brain glucose levels induced by natural arousing stimuli and intravenous cocaine: fueling the brain during neural activation. $J$ Neurophysiol 108: 1669-1684.

Kiyatkin EA, Ren S, Wakabayashi KT, Baumann MH, Shaham Y (2016). Clinically relevant pharmacological strategies that reverse MDMA-induced brain hyperthermia potentiated by social interaction. Neuropsychopharmacology 41: 549-559.

Kiyatkin EA, Wakabayashi KT (2015). Parsing glucose entry into the brain: novel findings obtained with enzyme-based glucose biosensors. ACS Chem Neurosci 6: 108-116.

Liu KJ, Hoopes PJ, Rolett EL, Beerie BJ, Azzawi A, Goda F et al (1997). Effects of anesthesia on cerebral tissue oxygen and cardiopulmonary parameters in rats. Adv Exp Med Biol 411: 33-39.

Laubie M, Schmitt H, Canellas J, Roquebert J, Demichel P (1974). Centrally mediated bradycardia and hypotension induced by narcotic analgesics: dextromoramide and fentanyl. Eur J Pharmacol 28: 66-75.

Lecrux C, Hamel E (2011). The neurovascular unit in brain function and disease. Acta Physiol (Oxf) 203: 47-59.

McLaughlin K (2017). Deadly chemistry. Science 355: 1364-1366.

Moon BU, de Vries MG, Cordeiro CA, Westerink BH, Verpoorte E (2013). Microdialysis-coupled enzymatic microreactor for in vivo glucose monitoring in rats. Anal Chem 85: 10949-10955. 
Nakao Y, Itoh Y, Kuang TY, Cook M, Jehle J, Sokoloff L (2001). Effects of anesthesia on functional activation of cerebral blood flow and metabolism. Proc Natl Acad Sci USA 98: 7593-7598.

Pattinson KT (2008). Opioids and the control of respiration. $\mathrm{Br} J$ Anaesth 100: 747-758.

Paxinos G, Watson C (1998). The Rat Brain in Stereotaxic Coordinates, 4th edn. Academic Press: San Diego.

Peng PW, Sandler AN (1999). A review of the use of fentanyl analgesia in the management of acute pain in adults. Anesthesiology 90: 576-599.

Schmidt CF, Kety SS (1947). Recent studies of cerebral blood flow and cerebral metabolism in man. Trans Assoc Am Physicians 60(1 vol): $52-58$.

Silver IA, Erecinska M (1994). Extracellular glucose concentration in mammalian brain: continuous monitoring of changes during increased neuronal activity and upon limitation in oxygen supply in normo-, hypo-, and hyperglycemic animals. J Neurosci 14: 5068-5076.

Solis E Jr, Cameron-Burr KT, Kiyatkin EA (2017a). Rapid physiological fluctuations in nucleus accumbens oxygen levels induced by arousing stimuli: relationships with changes in brain glucose and metabolic neural activation. Front Integr Neurosci 11: 9.

Solis E Jr, Cameron-Burr KT, Shaham Y, Kiyatkin EA (2017b). Intravenous heroin induces rapid brain hypoxia and hyperglycemia that precede brain metabolic response. eNeuro. (doi: https://doi.org/10.1523/ENEURO.0151-17.2017).

Suzuki J, El-Haddad S (2017). A review: fentanyl and nonpharmaceutical fentanyls. Drug Alcohol Depend 171: 107-116.

von Gunten CF, Bruera E, Pirrello RD, Portenoy RK (2010). New opioids: expensive distractions or important additions to practice? $J$ Palliat Med 13: 505-511.

Wade CL, Vendruscolo LF, Schlosburg JE, Hernandez DO, Koob GF (2015). Compulsive-like responding for opioid analgesics in rats with extended access. Neuropsychopharmacology 40: 421-428.

Willette RN, Doorley BM, Sapru HN (1987). Activation of cholinergic mechanisms in the medulla oblongata reverse intravenous opioid-induced respiratory depression. J Pharmacol Exp Ther 240: 352-358.

Windels F, Kiyatkin EA (2006). General anesthesia as a factor affecting impulse activity and neuronal responses to putative neurotransmitters. Brain Res 1086: 104-116.

Wise RA, Bozarth MA (1987). A psychomotor stimulant theory of addiction. Psychol Rev 94: 469-492.

Xu H, Li SJ, Bodurka J, Zhao X, Xi ZX, Stein EA (2000). Heroininduced neuronal activation in rat brain assessed by functional MRI. Neuroreport 11: 1085-1092.

Yeadon M, Kitchen I (1989). Opioids and respiration. Prog Neurobiol 33: 1-16.

Supplementary Information accompanies the paper on the Neuropsychopharmacology website (http://www.nature.com/npp) 